\title{
Gestión, tecnología, información \\ y comunicación como agentes de innovación en la educación
}

\author{
Fecha de recepción: 9 de agosto de 2011 - Aprobación: 14 de octubre de 2011
}

\section{Martha Inés Kammerer David - María PeÑa - Gusmary Chávez}

\section{Resumen}

El propósito del artículo es analizar la gestión de las TIC como agentes de innovación de cambios en la educación. Para ello, se realizó una revisión de carácter documental, desde el punto de vista metodológico bajo un paradigma global complejo, a fin de obtener reflexiones que permitan al personal de las organizaciones educativas manejar las tecnologías de la información y la comunicación (TIC). Por todas estas razones, es importante la comprensión fenomenológica de las ontologías del ser, entre las cuales integran diversas herramientas sociales. De allí, se enuncian lineamientos teóricos para la formación del personal docente y los estudiantes a fin de lograr una efectiva gestión en la práctica educativa, pretendiendo que dicha función esté a favor y sea apropiada por la comunidad educativa para el progreso de las organizaciones educativas, asimismo se presentan unas reflexiones finales.

\section{Abstract}

The purpose of this article is to analyze the management of ICT as agents of education change innovation. For this, we conducted a review of documentary character, from the methodological point of view under the complex global paradigm, in order to obtain insights that enable the staff of educational organizations to manage information and communication technologies. For all these reasons, it is important to understand the phenomenological ontologies of being, which integrate various social tools. From there, theoretical guidelines are set in order to train faculty and students to achieve effective educational practice management, seeking that function to favor the whole team in order for them to appropriate the progress of educative organizations as well as to present some final reflections.

\section{Palabras clave}

Gestión, tecnología, comunicación, innovación y educación.

\section{Key words}

Management, Technology, Communication, Innovation and Education.

\section{Introducción}

Las tecnologías de la información y comunicación han evolucionado en la organización de la enseñanza y el proceso de aprendizaje, trayendo consigo un conjunto de cambios e innovaciones. Por esto, siendo la educación pilar fundamental en la formación de los futuros ciudadanos para esta nueva sociedad del conocimiento, no puede quedar lejos de las incidencias de éstas ni del entorno social que se está configurando actualmente ante la presencia de las mismas. 
Evidentemente, las tecnologías de la información y la comunicación se están convirtiendo en un elemento clave en el sistema educativo, por lo tanto, la incorporación de éstas ha de hacerse con la perspectiva de favorecer los aprendizajes, facilitando los medios para sustentar el desarrollo de los conocimientos y de las competencias necesarias para la integración social.

En efecto, para que estas tecnologías estén verdaderamente al servicio del proceso de enseñanza aprendizaje, la tecnología debe estar acompañada de una eficiente gestión, involucrando la interacción de personas, recursos, y elementos de las organizaciones educativas.

En este sentido, se concibe la gestión de las TIC como un sistema de conocimientos prácticos relacionados con los procesos de creación, desarrollo, transferencia y uso de la misma.

Dicho de otro modo, la gestión tecnológica es un conjunto de decisiones asociadas a la adquisición, desarrollo y utilización, así como también la asimilación de tecnologías, tanto desde el punto de vista estraté-

\section{Reseña de autor \\ Martha Inés Kammerer David (Colombia) \\ Universidad de la Guajira \\ marthakammerer@gmail.com}

María Peña (Venezuela)

Universidad Rafael Belloso Chacin (URBE)

Profesora de la Universidad Pedagógica Experimental Libertador UPEL

lindamarina@urbe.edu

\section{Gusmary Chávez (Venezuela)}

Universidad Rafael Belloso Chacin (URBE)

Maestrante en Mercadeo, Licenciada en Comunicación Social, Mención audiovisual, Segunda Jornada de Investigación UPEL. Maracaibo.

gusmary18@hotmail.com gico como operacional, que se manejan con el fin de ofrecer una orientación acelerada a la innovación de una organización.

Sucede, que esta gestión e incorporación de las tecnologías presentes en las organizaciones de enseñanza-aprendizaje, potencie la transformación de las mismas, en ámbitos educativos más dinámicos, versátiles e innovadores. Por esta razón, se hace necesario presentar la importancia de las tecnologías en las organizaciones educativas.

\section{Consideraciones generales}

De igual manera, el proceso de gestión, así como el uso de la tecnologías mismas, sólo pueden darse con el dominio de habilidades y destrezas que constituyen las capacidades tecnológicas, facilitando todas las actividades involucradas en el desarrollo tecnológico. Estas actividades forman parte de los recursos tecnológicos, incorporando un conjunto de medios materiales (maquinarias, equipos, entre otros), además de distintos elementos inmateriales como la propiedad intelectual, las bases de datos y de conocimientos.

En términos generales, Ávalos, citado por Aronica y Peretti (2006: 38) señala que los procesos de gestión tecnológica en las organizaciones de cualquier tipo, involucran funciones básicas como: identificación, evaluación y selección de tecnologías, desagregación de paquetes tecnológicos, construcción y puesta en marcha de sistemas productivos, uso, asimilación, adaptación, mejoramiento, generación y comercialización de nuevas tecnologías. Asimismo, los agrupa en tres ámbitos: el de la adquisición, uso y realización de actividades de investigación y desarrollo, en los 
que ubica algunas de las funciones de gestión tecnológica antes señaladas.

En este sentido, se entiende el proceso de gestión tecnológica como diverso y dinámico, en cuyo desarrollo se reconoce la existencia de ciertos patrones estructurales que constituyen la clave para comprender su progreso, pero también para atender las singularidades que le son inherentes. Por tanto, la aceptación implícita y explícita por parte de las instituciones, para gestionar e incorporar las TIC a la educación como recurso y eje de los nuevos aprendizajes, hace que no se pueda tomar a la ligera dicho proceso de adquisición, utilización y asimilación de las tecnologías de la información.

Por otra parte, en el proceso de gestión tecnológica se abarcan diversas acciones, así lo señalan Hidalgo, y col., citados por Amador y Márquez (2009: 18), al mencionar la identificación, evaluación, selección, adquisición, asimilación y utilización de las tecnologías requeridas como las actividades que caracterizan el mismo, y cada organización debe ajustar estos procedimientos a sus respectivas necesidades e intereses.

Cabe resaltar, que los procesos de gestión tecnológica no terminan cuando ésta es adquirida e incorporada a los proyectos que se ejecuten, generalmente es necesario evaluar su uso o proceder a optimizaciones (limitadas por las condiciones legales de su adquisición). Por último, en algún momento habrá que tomar la decisión de retirarla por considerarla obsoleta u otros motivos.

Por consiguiente, la gestión tecnológica busca integrar el proceso de cambio tecnológico con los aspectos estratégicos y operativos de control, además, con la toma de decisiones de la empresa. En consecuencia, este proceso en la organización significa la aplicación de un conjunto de prácticas que permiten establecer estrategias en materia tecnológica congruentes con los planes de acción. En el ambiente educativo, éste debe asumirse en los propósitos y políticas tecnológicas para la adquisición, uso y creación de tecnología, apoderándose de la innovación como eje de las estrategias de desarrollo dentro de las instituciones educativas.

La gestión es la acción que permite planificar, organizar, dirigir o ejecutar; así como controlar y evaluar lo realizado en cualquier situación; por ello, Mora, citado por Ochoa y col (2007: 4), la define como "el conjunto de diligencias que se realizan para desarrollar un proceso o para lograr un producto determinado". Se asume como la dirección de las actividades necesarias para hacer que las cosas funcionen, con la capacidad para generar procesos de transformación de la realidad; y además como una función institucional global e integradora de todas las fuerzas que conforman una organización, por lo tanto la gestión enfatiza en la dirección y en el ejercicio del liderazgo.

En ese orden de ideas, se considera que la gestión es un proceso que comprende determinadas funciones y actividades laborales que los gerentes deben llevar a cabo mediante objetivos, lo cual permite que las instituciones educativas sepan organizarse y saber con qué se cuenta; de allí, puede ser emprendida por una persona o por más para coordinar las actividades laborales de otros, con la finalidad de lograr resultados de alta calidad.

Haciendo una analogía con este concepto de la gestión en la administración, la gestión de las tecnologías de la información y la comunicación se puede definir como la aplicación de los conocimientos científi-
Lagestión es la acción que permite planificar, organizar, dirigir o ejecutar; así como controlar y evaluar lo realizado en cualquier situación. 
cos, según lo expresa Benjumea (2006: 50), organizados en torno a tareas prácticas, es decir, la gestión es la aplicación de la ciencia. También puede entenderse como proceso de concepción, diseño y fabricación de los instrumentos que dan soluciones a problemas o necesidades presentadas en el entorno.

De igual forma, la tecnología puede concebirse de acuerdo con criterios a nivel mundial, según Benjumea (2006: 50), como un fenómeno cultural de orden interdisciplinario que posibilita la transformación de la naturaleza y la cultura por el hombre y que es susceptible de ser estudiada, comprendida y mejorada por las generaciones presentes y futuras. De este modo, la tecnología es proceso, arte y ciencia, tiene relación directa con lo que hace el hombre y sus necesidades, buscando con ella crear, innovar para satisfacer sus expectativas e intereses, por supuesto contribuyendo con el desarrollo y el progreso de éste en la sociedad.

De esta manera, las TIC se refieren, como lo expresa Rosario (2005: 1), "al conjunto de tecnologías que permiten la adquisición, producción, almacenamiento, tratamiento, comunicación, registro y presentación de informaciones, en forma de voz, imágenes y datos contenidos en señales de naturaleza acústica, óptica o electromagnética". De este modo, las TIC, se fundamentan en la aplicación de la ciencia para la optimización de procesos de información y comunicación, con base en las telecomunicaciones e informática, planteados desde la perspectiva de la construcción y socialización del conocimiento.

En esta perspectiva, la innovación docente establece novedades que mejoran el proceso formativo incidiendo en las formas de construcción del conocimiento, en la configuración de nuevos entornos de enseñanza-aprendizaje y en la transformación de la cultura escolar docente. La incorporación de las TIC al entorno educativo puede ser vista, entonces, como un proceso de innovación, al generar cambios significativos en el desarrollo del trabajo pedagógico.

\section{A. Lineamientos teóricos}

Se evidencia la necesidad de formación para el desarrollo de competencias que permitan al docente, el manejo de las tecnologías de la información, se enuncian lineamiento teóricos para la formación del personal que labora en las escuelas básicas requeridas a fin de lograr una efectiva gestión de las mismas en la practica educativa, pretendiendo que dicha formación esté a favor de todo el equipo involucrado y que la estructura organizacional de los centros educativos de las escuelas básicas del Municipio Maracaibo 1, en Venezuela, se apropien de estos lineamientos y del sentido de presentar viabilidad en las mismas.

El propósito de estos lineamientos es brindar directrices teóricas que sirvan de base para generar el cambio de manera conceptual, procedimental y actitudinal, convirtiéndose en el punto de partida para futuras acciones de formación del personal directivo y docente de los centros educativos, de manera que identifiquen los elementos que constituyen las competencias para darle aplicación a las herramientas tecnológicas, asumiendo los conocimientos necesarios para crear e innovar, de manera que puedan afrontar el cambio científico, tecnológico y de formación que caracteriza a esta sociedad $y$, por ende, al sistema educativo. 
Por lo tanto, es importante dar información suficiente para que los docentes realicen trabajos usando la tecnología, de igual manera resulta clave orientar al personal en el uso de la misma, promover el quehacer (consecución de metas) de los directivos y los docentes mediante la aplicación de las TIC y, y el uso de redes digitales como medio socioeducativo de comunicación interactiva.

\section{Elementos de gestión de las tecnologías de información y comunicación}

Ante la gestión e incorporación de las TIC en la enseñanza, es necesario contemplar algunos elementos relacionados con este proceso. Al respecto, Cabero (2007: 36) señala algunos aspectos a considerar: "el alumno, el profesor o docente, las herramientas, la metodología, los equipos, las funciones, entre otros", que como se observan, la gestión involucra tanto los recursos humanos como los materiales para poder desarrollar el proceso educativo.

Resulta importante comprender la dinámica presente en los elementos antes mencionados para la efectiva aplicación y gestión de las tecnologías en el sector educativo, sin olvidar que no son sólo docentes y estudiantes son los protagonistas significativos del proceso, sino también, todos y cada uno de los medios, sujetos e instrumentos que permiten llevar a cabo dicha aplicación.

Marqués (2004: 93), desde una perspectiva sistémica, plantea que el uso y la organización de los recursos de un centro exige ordenar las relaciones que se pueden dar entre todos los elementos que intervienen en la gestión, especialmente cuando se pretende la aplicación didáctica de todos los recursos. De este modo, distingue tres tipos de recursos: a) los materiales (infraestructura física, aparatos tecnológicos y materiales curriculares), b) los humanos (profesores, alumnos, directivos y coordinadores) y c) los funcionales (estrategias y herramientas), los cuales confluyen de manera dinámica en el proceso educativo.

Desde este punto de vista, Galván (2005: 18) también señala que los elementos presentes en la gestión de las tecnologías, al interior de las instituciones educativas, son los recursos humanos, los recursos materiales y de infraestructura, y los elementos funcionales; así coincidiendo con el autor, se evidencia la necesidad de contar con éstos cuando se requiere gestionar las TIC en cualquier entorno organizacional.

\section{Elementos tecnológicos}

Para el desarrollo de la gestión de las TIC, es importante evaluar con qué equipos y materiales se cuenta, porque de no ser por el uso de ellos, sería imposible ejecutar las operaciones. Por su parte, Galván (2005: 33), denomina que las aulas informáticas, las salas de estudio multiuso con ordenadores y conexión a Internet, la pizarra digital, la intranet, la plataforma, las Web del centro y las aulas de audiovisuales son recursos materiales y de infraestructura presentes en el manejo de tecnologías dentro de las instituciones educativas.

Por otra parte, los elementos materiales que intervienen en la gestión de las TIC en los centros educativos comprenden varios tipos, a saber: equipos tecnológicos, infraestructura física, redes, herramientas e instrumentos que facilitan y favorecen la acción didáctica; por ello, la riqueza y variedad de los recursos que en la actuali- 
dad puedan ser empleados en la educación, constituyen una oportunidad de especial significado para las escuelas que desean alcanzar la calidad a través de la incorporación de las tecnologías.

De manera semejante, Marqués (2004: 65), afirma que en la organización de los recursos tecnológicos de un centro, se pueden distinguir tres tipos de elementos materiales: la infraestructura física, los aparatos tecnológicos y los materiales curriculares, los cuales agrupa y define el autor, expresando que todos estos elementos, en conjunto, son necesarios para la ejecución de las operaciones.

Por una parte, el autor antes mencionado enuncia que en la infraestructura física se considera el espacio disponible para el uso y el almacenamiento de los recursos, como aulas telemáticas, rincones, almacenes; asimismo, las instalaciones, iluminación, conexiones eléctricas y en red, ventilación, sistemas de seguridad; y por ultimo, los materiales complementarios, entre éstos, mesas, armarios, sillas, estantes y otros.

Seguidamente, otros de los elementos presentes en la gestión de las TIC, son los aparatos tecnológicos, los cuales son equipos necesarios para poder usar los materiales curriculares que se presentan en soporte tecnológico. El punto de partida para la integración éstos son los centros educativos, obviamente la disponibilidad de recursos tecnológicos, como ordenadores, impresoras, conexiones telemáticas, módems, proyectores, reproductores de sonidos, cámaras fotográficas y otros hardwares informáticos, debidamente ubicados e instalados, y con un adecuado sistema de mantenimiento.

Por ultimo, siguiendo la opinión del mismo autor, los materiales curriculares, que conjuntamente con sus guías pedagógicas, incluyen la información, contenidos programáticos y, en algunos casos, también proponen las actividades didácticas que facilitan el proceso de enseñanza y los aprendizajes basados en tecnologías para los estudiantes, sin olvidar que su utilización requiere el empleo de algunos aparatos tecnológicos.

En líneas generales, la adquisición de los equipos tecnológicos multimedia, así como la disponibilidad de redes con capacidad y velocidad adecuada, es cuestión primordial a contemplar en este aspecto. No obstante, los equipos evolucionan con gran rapidez apareciendo permanentemente en el entorno nuevos equipos que instigan a la actualización, y resultando clara e innegablemente que la utilidad de las tecnologías de la información y la comunicación son recursos para la enseñanza, pues propician el uso de herramientas innovadoras como el correo electrónico, el chat, las pizarras electrónicas, entre otros, dentro del entorno educativo.

\section{Elementos humanos}

De igual manera, se supone que para el desarrollo de la gestión de las tecnologías de información y comunicación, respecto a la innovación educativa, se debe partir de los elementos humanos o personales para la gestión y organización de los recursos tecnológicos de un centro educativo, los cuales son para Marqués (2004: 70), los siguientes:

Los profesores y estudiantes. Ellos utilizan los recursos audiovisuales e informáticos con diferentes finalidades: a) para preparar clases o b) como material complementario de las exposiciones orales, 
también c) como un medio alrededor del cual desarrollan una actividad concreta de aprendizaje, d) o bien como herramienta para realizar diversas actividades (p.ej. cálculo, apuntes, búsqueda de información y redacción de trabajos).

El equipo directivo. La aptitud de los miembros del equipo directivo, respecto a los medios tecnológicos, resulta de capital importancia para asegurar una buena gestión de recursos y el buen funcionamiento de las aulas. Una dirección innovadora facilita la incorporación y la coordinación de su uso. Asimismo, promueve actuaciones formativas e informativas dirigidas al profesorado. Periódicamente evalúa el funcionamiento del servicio y el presupuesto del centro, los gastos necesarios para el mantenimiento de los equipos y la compra de nuevos materiales curriculares.

El personal auxiliar no docente, son las personas que realizan algunos trabajos relacionados con la conservación de las aulas y el mantenimiento de los recursos tecnológicos: conserjes, personal de limpieza, encargados del cuidado y/o técnicos de mantenimiento de los equipos.

Los coordinadores de los recursos tecnológicos, son quienes deben proporcionar soporte y capacitación tanto al estudiante como al profesor para superar los problemas que se les presenten al aplicar las nuevas tecnologías, así como también son los llamados a impulsar y promover la innovación dentro de los procesos. Para que ellos puedan realizar su labor, necesitan disposición horaria, apoyo de dirección, aceptación por parte de los docentes, conocimiento de los aparatos tecnológicos y su utilización, así como también preparación pedagógica.
En ese mismo orden de ideas, Galván (2005: 111) expone que los elementos o recursos humanos que intervienen en la gestión son el profesorado, los alumnos, el equipo directivo, los coordinadores, el personal auxiliar y técnico especial presente en las instituciones educativas. De esta manera, forman parte de este conjunto, las personas que propicien la integración, organización y utilización de los recursos tecnológicos en la práctica pedagógica.

Evidentemente, se hace necesario que los sujetos estén más capacitados para movilizar, gestionar, manejar y utilizar las nuevas herramientas tecnológicas en el proceso de enseñanza-aprendizaje, pues como se ha expresado, son estos recursos los vinculadores directos de los actores involucrados con los avances tecnológicos generados, es decir, los hacen partícipes del proceso de gestión tecnológica concebido desde el entorno educativo.

Cabe destacar, que los elementos humanos son imprescindibles, porque de no ser por ellos, las tecnologías de la información y la comunicación no podrían incorporarse al proceso educativo, de allí que sea necesaria la formación y actualización constante de conocimientos y habilidades para alcanzar resultados exitosos dentro de la práctica pedagógica.

\section{Elementos funcionales}

Otro aspecto que debe tomarse en cuenta, en la gestión de las TIC, son los elementos funcionales. Al respecto, Marqués (2004: 7) señala que estos son :

El horario y la normativa de uso de los recursos son elementos indispensables para la gestión tecnológica de un centro, el horario de utilización de las instalaciones y 
de los materiales, elaborado según las peticiones de los profesores, es clave y para ello es imperioso que se coloque en un lugar bien visible. También resulta imprescindible la normativa de uso de éstos, porque determinará las prioridades para utilizar las instalaciones y sus equipos, las normas de aulas, los procedimientos a seguir para recoger los materiales e informar las incidencias, de igual modo, las funciones y las responsabilidades de los coordinadores tecnológicos.

El inventario y mantenimiento de los recursos para cada uno de los aparatos tecnológicos, es muy importante y, para ello, será muy útil configurar una ficha de inventarios que recogerá sus características básicas y la de los materiales. También, sobre cada aparato, debe existir la fecha de las diferentes modificaciones e incidencias de su reparación y mantenimiento. De esta manera, los coordinadores pueden revisar estas anotaciones cada día, para poder controlar e iniciar inmediatamente las acciones oportunas para el arreglo de los mismos, situación que debe ser controlada con exactitud para evitar perdida de tiempo en cuanto a la administración de las actividades con estos equipos.

Sesiones informativas y formativas. Estas sesiones se pueden realizar con frecuencia, con la finalidad de mostrar a los profesores cómo se pueden utilizar los diversos recursos tecnológicos disponibles, al mismo tiempo informar de los materiales adquiridos por el centro educativo e intercambiar experiencias sobre el aprovechamiento didáctico de los mismos.

Servicio y asesoramiento continuo para facilitar tanto a profesores como a estudiantes, la utilización de los recursos tec- nológicos, sería conveniente que en todo momento de los problemas concretos que surjan, de allí, la importancia de contar con personas que orienten y asesoren el trabajo de manera que se alcancen los objetivos planificados.

Atendiendo estas consideraciones, es importante que se desarrollen funciones y pautas orientadoras que administren el uso de los recursos tecnológicos y su funcionamiento, para garantizar así la utilización adecuada por parte de todos los actores involucrados en la comunidad educativa. Sin duda, la organización del grupo, recursos tecnológicos y de la institución misma, garantiza la eficacia de la gestión de las tecnologías de información y comunicación en el campo educativo, favoreciendo e impulsando la innovación.

Cabe destacar que, Ochoa y col (2007: 264) plantean que para darle funcionamiento a la tecnología, se precisa incluir las siguientes actividades; seguimiento, análisis y prospectiva tecnológica, planificación del desarrollo tecnológico, diseño de estrategias, identificación, evaluación y selección de tecnologías, adaptación e innovación, negociación, adquisición y contratación, comercialización de tecnologías de la empresa, planeamiento, financiación, selección y capacitación de asesores y operadores, gestión de proyectos de investigación y desarrollo, así como suministro y evaluación de información técnica.

\section{Adquisición de tecnologías}

En esta perspectiva, Ávalos, citado por Aronica y Peretti (2006: 236), presenta la adquisición de tecnología con un papel importante en la acumulación de capacidades referidas a ésta, por ello se le asigna el 
propósito general de convertir los métodos de compra de tecnología en procesos que permitan una verdadera transferencia de este recurso; orientando la gestión para adquirir las capacidades tecnológicas, y así usarla adecuadamente, adaptarla y mejorarla, más que a adquirir una capacidad productiva. Además para este autor, las etapas globales de este proceso incluyen: la búsqueda de información, selección, evaluación, negociación y adopción de la misma, éstas se explican a continuación:

Selección. Esta actividad resulta clave en la etapa de adquisición porque abarca la selección de tecnología y de proveedores; además esta vinculada a los proyectos de inversión, debido a los requerimientos tecnológicos del mismo. Al efectuarse la selección debe considerarse la fase en la que se encuentra la tecnología (experimentación, desarrollo madurez, decadencia) dentro de la organización, obsolescencia de las mismas, inadecuación del paquete tecnológico con respecto a la empresa compradora, revisión de resultados del paquete a adquirir en otras partes, evaluación de los costos, las posibilidades de incorporar capacidad tecnológica local asociada al paquete a adquirir, así como las implicaciones presentes en el ambiente interno de la institución.

Negociación y adopción de tecnología. Comprende la etapa de entendimiento con un determinado proveedor para establecer precio y condiciones de la compra - venta, en este proceso, la institución debe evaluar previamente sus capacidades tecnológicas, designación y alternativas en el mercado. Adicionalmente, cabe destacar la importancia de considerar otros factores influyentes en este proceso de negociación, tales como el comprador, posición en el mercado, sector en el que se desempeña, conocimiento que tiene el proveedor, infraestructura científico - tecnológica, el tipo de tecnología, su modernidad, naturaleza y complejidad.

Según Cegesti (2005: 72), una empresa tiene que hacer de las tecnologías un recurso necesario, sea por medio de un esfuerzo interno de desarrollo o mediante la adquisición externa, dicho de otro modo, debe aprovecharse de estas herramientas para avanzar hacia la innovación y la transformación.

De igual manera, las tecnologías se crearon para facilitar las operaciones en las organizaciones, pues son recursos y herramientas que se utilizan para agilizar el trabajo del hombre. Ávalos, citado por Aronica y Peretti (2006: 207), destacan también, la necesidad de hacer gestión tecnológica durante el uso de la tecnológica, desarrollando estrategias diseñadas para convertir la experiencia en producción, mantenimiento y procesos de aprendizaje. Es decir, que la gestión tecnológica en el uso de la tecnología, tiene el objetivo de usar la experiencia como fuente de aprendizaje consciente, racionalizándola para adquirir conocimientos complementarios a los que se recibieron del proveedor de tecnología.

Por lo tanto, la tecnología debe integrarse a los procesos de aprendizaje planeados, organizados y desarrollados para una asimilación plena de la misma, y lograr así una explotación de su máximo potencial, con la optimización, adecuación y adaptación a las condiciones cambiantes del ambiente donde opera. Una tecnología contemplada fuera del marco en el que ha de actuar y sin relación con las necesidades concretas de los actores involucrados en el
Según Cegesti (2005: 72), una empresa tiene que hacer de las tecnologías un recurso necesario, sea por medio de un esfuerzo interno de desarrollo o mediante la adquisición externa, dicho de otro modo, debe aprovecharse de estas herramientas para avanzar hacia la innovación y la transformación. 
proceso educativo conlleva a una utilización reducida tanto en el aprovechamiento como en el desarrollo de las cualidades que ésta pueda otorgar al proceso educativo.

Al respecto, Navarro (2002: 37) plantea que en el ámbito escolar, será necesario reflexionar sobre el uso tecnológico en los centros y en las aulas, del mismo modo es necesario preguntarse ¿cómo van a intervenir en el proceso educativo, los medios tecnológicos?, ¿cuál va a ser su intervención a nivel curricular? A propósito, Peñafiel, citado por Navarro, afirma que el uso de éstos debe estar fundamentado en el siguiente principio: "conocer el valor que supone incluir las innovaciones tecnológicas en el proceso de transformación de la organización educativa para la sociedad".

Sin embargo, lo más importante de los medios es la capacidad de éstos para poner a los estudiantes en contacto continuo e inmediato con experiencias de aprendizaje. Para ello, se debe adecuar los recursos disponibles a las necesidades de los mismos y a las características personales de la propia institución educativa, además poseer criterios para seleccionar aquellos medios valorando sus ventajas e inconvenientes, así como las dificultades que plantea ponerlos en práctica. Por último, es preciso tener una perspectiva sobre la función pedagógica que desempeña aspectos: organizativos, metodológicos y criterios de evaluación.

Efectivamente, con el uso de las tecnologías hay un nuevo panorama educativo que obliga a reflexionar sobre la necesidad de replantearse los cambios en la metodología, las estrategias de enseñanza y los sistemas de evaluación utilizados tradicionalmente. En este nuevo discurso sobre el acto educativo, y los procedimientos de intervención didáctica, es necesario reflexionar acerca de cuál es el lugar que ocupa la tecnología.

En este sentido, también habrá que plantearse la siguiente pregunta: ¿cuáles son las posibilidades que tienen los usuarios para acceder a los medios informáticos dentro y fuera de los centros? . Las tecnologías de la información y la comunicación no tienen en sí mismas, el poder de producir cambios en las realidades educativas, pero no hay duda que utilizándolas efectivamente pueden ser de gran ayuda. Evidentemente, estos recursos son medios poderosos que utilizados adecuadamente logran convertirse en grandes aliados para el logro de los fines educativos; de allí, la necesidad de contar no sólo con los equipos necesarios, sino también con el personal preparado para su utilización.

Por esta razón, las tecnologías adquiridas deben ser asimiladas por parte del personal de la institución con la finalidad de adaptarlas a sus condiciones específicas, a través de la generación de información técnica y de nuevos conocimientos que contemplen el paquete adquirido, permitiéndole responder a las exigencias del ambiente con un mejor uso de la capacidad instalada. En este proceso, el conocimiento se convierte en un elemento central, donde su potencial se valora con relación a los aportes que otorguen a la transformación mejorada de las tecnologías.

Primera (2000: 40), señala que el proceso de asimilar las tecnologías debe estar constituido por una infraestructura educativa, informacional, comunicacional, operacional y de ingeniería para responder a las decisiones relacionadas con el mismo, a la continuidad de los programas, la disponibilidad complementaria y el apoyo financiero que reciben los condicionantes; 
todas estas variables favorecen el proceso de adaptación, pues generan un cúmulo de experiencias que conjuntamente con las estrategias conforman el sistema de aprendizaje de la organización.

Por su parte, Ochoa y col. (2007: 265), expresan que asimilar significa que una vez realizados los pasos anteriores, es posible actuar en la explotación sistemática del potencial tecnológico mediante programas de capacitación, documentación de tecnologías de la empresa, desarrollo de aplicaciones derivadas de tecnologías genéricas y gestión eficiente de recursos.

Por lo tanto, se asume, necesariamente, que para poder llevar el proceso de la gestión tecnológica adelante, el docente debe formarse, y aprender en qué consiste adquirir información, utilizarla eficazmente para darle respuesta a los distintos problemas que surgen en el aula y el plantel, asimilándola como una herramienta básica de carácter administrativo y académico presente en el medio educativo.

\section{Reflexiones finales}

$\mathrm{Al}$ analizar la gestión de las tecnologías de la información y comunicación como agentes de innovación y cambio en la educación básica, se dan las siguientes conclusiones:

Se evidencia que el personal de algunas organizaciones educativas, no se interesan por incorporar la utilización de las tecnologías, así como se observa el poco interés que muestran en apropiarse de la gestión administrativa, en darle promoción o en adquirir nuevas tecnologías.

De igual manera pasa con el personal docente de las escuelas básicas que no se motivan, aunque su culminación laboral esté cerca, a usar Internet Se pudo cono- cer que existe desinterés en el manejo del correo electrónico, el chat, lo webquests y weblog, entre otros.

Por todas estas razones, la gestión y el manejo de las TIC, para el personal, no es innovador, y presentan justificación de manera oral, pues no ven las TIC como agente de cambio; sin embargo, los estudiantes están interesados en que las organizaciones educativas se incorporen y promuevan las tecnologías como eje fundamental para la competencia y desarrollo del conocimiento, y por ende contribuir al desarrollo del país.

Por consiguiente, la formación y comunicación se deben considerar en las instituciones educativas, así:

Promoviendo un plan de formación del personal, partiendo de la alfabetización en el área informática y avanzando hacia niveles de mayor complejidad.

Agrupando a los docentes según su nivel de formación y destreza en el uso de las tecnologías.

Ofreciendo equipos tecnológicos para el uso del personal.

Programando actividades de formación que permitan la integración de la tecnología a las actividades curriculares de los docentes.

Promoviendo espacios para el personal docente para que utilicen las herramientas básicas del computador.

Enseñando la aplicación de las tecnologías de la información y la comunicación como herramientas para la transformación del aprendizaje.

Orientando la formación del personal que incorpore las tecnologías de la información y la comunicación a la actividad educativa permanente. 
Promoviendo la formación del personal a través de grupos de trabajo para el estudio de recursos tecnológicos.

Entrenando a los docentes en el nivel usuario para que utilicen los programas ofimáticas elementales.

Promoviendo al docente hacia el nivel avanzado cuando éste haya adquirido las habilidades básicas para el manejo de las tecnologías de la información y la comunicación.
Reconociendo al docente cuando domina el uso de las tecnologías de la información y la comunicación, considerándolo en el nivel de experto, en el uso de las tecnologías, para que facilite a otros docentes sus conocimientos.

Y finalmente, propiciando un plan de integración en los estudiantes, con el propósito de ir avanzando en el uso, capacitación y dominio de las nuevas tecnologías en la escuela básica.

\section{Bibliografía}

1. Amador y Márquez (2009). Un modelo conceptual para gestionar la tecnología en la organización. En Revista Espacios, vol. 30 (1). Caracas.

2. Aronica y Peretti (2006). La gestión tecnológica de las empresas del sector manufacturero del plástico en el Estado Zulia. Tesis doctoral, Universidad D. Rafael Belloso Chacín. Maracaibo.

3. Benjumea J. (2006). Educación en tecnología donde comienza el futuro. Federación Internacional de Fe y Alegría. Caracas.

4. Cabero J. (2007). Nuevas tecnologías aplicadas a la educación. McGraw Hill. Madrid.

5. Galván A. (2005). Organización y gestión de un centro TIC. En Revista Digital Investigación y Educación, vol.3 (20).

6. Cegesti (2005) Manual de transferencia y adquisición de tecnologías sostenibles. San José, Costa Rica.

7. Marqués P. (2004). La organización de los recursos tecnológicos de un centro. Las aulas de recursos. Versión electrónica. Barcelona - España.

8. Navarro M. (2002). La utilización de la tecnología, otro modo de atender a la diversidad. Universidad de Sevilla Píxel-Bit. Versión electrónica.

9. Ochoa y col (2007). Innovación, tecnología y gestión tecnológica. Versión electrónica, vol.16 (4).

10.Paredes A. (2004). Gestión de tecnologías de información y comunicación: soportes para la innovación en las organizaciones inteligentes. Revista de Ciencias Sociales, vol.10 (3). Versión electrónica.

11.Primera N. (2000). Gestión de tecnologías de información en bibliotecas universitarias. Tesis doctoral. Universidad Dr. Rafael Belloso Chacín. Maracaibo.

12. Rosario J. (2005). La tecnología de la información y comunicación (TIC). Su uso como herramienta para el fortalecimiento y el desarrollo de la educación virtual. Versión electrónica. 